\title{
Role of gamma probes in performing minimally invasive parathyroidectomy in patients with primary hyperparathyroidism: optimization of preoperative and intraoperative procedures
}

\author{
Domenico Rubello, Andrea Piotto ${ }^{1}$, Dario Casara, Pier Carlo Muzzio ${ }^{2}$, Brahm Shapiro ${ }^{3}$ and Maria Rosa Pelizzo ${ }^{1}$ \\ Nuclear Medicine Service, Department of Radiotherapy, Regional Hospital of Padua, Padua, Italy, ${ }^{1}$ Department of Clinical Surgery and \\ ${ }^{2}$ Oncological Radiology Service, University of Padua, Padua, Italy and ${ }^{3}$ Nuclear Medicine Service, Department of Radiology, \\ Michigan University Medical Center, Ann Arbor, Michigan, USA
}

(Correspondence should be addressed to D Rubello, Servizio di Medicina Nucleare 2, Dipartimento di Radioterapia, Azienda Ospedaliera di Padova via Giustiniani 2, 35100, Padua, Italy; Email: domenico.rubello@sanita.padova.it)

\begin{abstract}
Objective: In the last decade, surgery of primary hyperparathyroidism (HPT) due to a solitary adenoma has moved on from the traditional wide bilateral neck exploration (BNE) to more limited approaches such as unilateral neck exploration and minimally invasive parathyroidectomy.

Design: To define the role of intraoperative gamma probe and injection of a low ${ }^{99} \mathrm{~m}$ Tc-MIBI dose in performing minimally invasive radio-guided surgery (MIRS) in HPT patients with a solitary parathyroid adenoma.

Methods: From September 1999 to July 2002, 214 patients with primary HPT entered the study. All patients were preoperatively investigated by a ${ }^{99} \mathrm{~m}$ Tc-pertechnetate/MIBI subtraction scan and high-resolution neck ultrasound. The intraoperative technique we developed differs from other previously described techniques being based on the injection of a low (37 MBq) MIBI dose in the operating theatre a few minutes before the beginning of intervention.

Results: On the basis of scan/ultrasound findings 147 patients were selected for a MIRS and 144 of them $(98 \%)$ were successfully treated by this approach: a solitary parathyroid adenoma was removed through a small $2-2.5 \mathrm{~cm}$ skin incision with a mean operative time of $35 \mathrm{~min}$, and a mean hospital stay of 1.2 days. In the other 67 patients with scan/ultrasound evidence of concomitant nodular goiter $(n=45)$ or multi-gland disease $(n=13)$ or with a negative scan $(n=9)$, the gamma probe was utilized during a traditional BNE. A low $37 \mathrm{MBq}$ MIBI dose proved to be sufficient to perform a MIRS; moreover it delivered to the patient and surgeon a low, negligible, radiation exposure dose. Conclusions: The combination of a ${ }^{99 m}$ Tc-pertechnetate/MIBI subtraction scan and neck ultrasound appears to be an accurate imaging protocol in selecting primary HPT patients as candidates for a MIRS. A MIBI dose as low as $37 \mathrm{MBq}$ injected in the operating theatre just before the start of surgery appears to be adequate to perform radio-guided surgery.
\end{abstract}

European Journal of Endocrinology 149 7-15

\section{Introduction}

The traditional approach to patients with primary hyperparathyroidism (HPT) consists of bilateral neck exploration (BNE) with the aim of visualizing all parathyroid glands and removing the enlarged ones (1). Several surgical protocols also include biopsy of the normal-sized glands in order to discover possible glandular hyperplasia (2). BNE has been shown to have a success rate as high as $95 \%$ in the hands of experienced surgeons (1). In surgical centers where BNE is routinely performed poor clinical value is usually assigned to preoperative localizing imaging, which is preferably reserved for patients with persistent/recurrent HPT before re-operation (3). It appears difficult to propose an alternative surgical approach able to further improve the efficacy of BNE. However, some considerations can be made: (i) without preoperative imaging, the parathyroid glands localized in orthotopic sites are easily detected intraoperatively - conversely, the ectopic glands can be missed; (ii) some morbidity (hypoparathyroidism and laryngeal nerve injury) is associated with BNE, especially when biopsy of normal-sized glands is applied or when re-operation is required for persistent/recurrent $\operatorname{HPT}(2,4)$; and (iii) cosmetic results are less than optimal. Furthermore, 
since primary HPT is caused by a solitary adenoma in about $85 \%$ of all cases, BNE is felt by many surgeons to be overtreatment in the majority of these patients. For these reasons, parathyroid surgery has progressively moved towards some type of limited neck exploration. The first attempt was made in the early 1980s by Tibblin et al. (5) who proposed a unilateral neck exploration (UNE) based on parathyroid adenoma removal and biopsy of the ipsilateral parathyroid gland to discover possible hyperplasia. In the 1990s UNE evolved towards even more limited surgical approaches: the endoscopic (6) and the radio-guided (7) minimally invasive parathyroidectomy. Two main factors strongly facilitated the development of minimally invasive surgery of solitary parathyroid adenomas. First, the significant improvement achieved in scintigraphic examination after the introduction of MIBI into clinical practice in the late 1980s thanks to Coakley (8). Secondly, the availability of assays for quick parathyroid hormone (PTH) measurement in the operating theatre: it has been demonstrated that a $>50 \%$ decrease of quick PTH levels after the removal of all hyperfunctiong parathyroid tissue is a strong predictor of complete disease remission (9). Moreover, the worldwide diffusion of probes as intraoperative devices during radio-guided surgery, especially sentinel node biopsy of melanoma and breast cancer patients, further encouraged the development of minimally invasive radio-guided surgery (MIRS) in HPT patients with a solitary parathyroid adenoma.

In the present study, following some preliminary papers from our center (10-13), we report our experience on probe-guided parathyroidectomy obtained in a large series of 214 consecutive primary HPT patients. The study is focused on optimization of the radio-guided technique and on definition of clinical indications for MIRS.

\section{Patients and methods}

From September 1999 to July 2002, 214 consecutive patients affected by primary HPT were enrolled in the study. Biochemical diagnosis of primary HPT was obtained in all patients. There were 128 females and 86 males, mean age 53.5 years (range 27-81 years). The reasons that led the physician to investigate calcium/phosphate metabolism were as follows: bone pain suggesting osteoporosis in $78 \%$ of cases, renal stones in $16 \%$, fatigue and neuro-muscular symptoms in $14 \%$, hypertension in $7 \%$, gastrointestinal symptoms in $4 \%$ (two or more of the above symptoms were contemporaneously present in one-third of patients); $12 \%$ of patients were asymptomatic and hypercalcemia was incidentally discovered during a routine blood sample analysis. Twenty-seven patients had previously undergone neck surgery for a thyroid nodular goiter or primary HPT in another surgical center.
The preoperative localizing imaging work-up consisted of a double-tracer ${ }^{99 \mathrm{~m}_{\mathrm{T}} \text {-pertechnetate/MIBI }}$ subtraction scan combined with high-resolution neck ultrasound (14). In cases of congruent scan/ultrasound results (both positive or negative) imaging was terminated, while in some cases of discrepancy (scan-positive and ultrasound-negative) for the presence of an enlarged parathyroid gland in the neck or with planar scintigraphy showing an ectopic parathyroid gland, the imaging protocol was completed by tomographic (single photon emission computed tomography (SPECT)) acquisition ( $n=36$ patients) in order to better define the depth of the parathyroid enlargement. SPECT was performed immediately after planar scintigraphy and ultrasound, thus commencing acquisition 50-60 min after MIBI injection. In this way the same MIBI dose was used for both planar scintigraphy and SPECT.

Patients were selected for MIRS on the basis of the following criteria: (i) scan evidence of a solitary parathyroid adenoma, (ii) a clear MIBI uptake in the parathyroid adenoma, (iii) absence of concomitant MIBI-avid thyroid nodules, (iv) no history of familial HPT or multiple endocrine neoplasia (MEN), and (v) no history of neck irradiation. One hundred and forty-seven patients met these inclusion criteria (68.7\% of all cases).

Sixty-seven patients were excluded from MIRS due to: (i) evidence of concomitant MIBI-avid thyroid nodules in 45 patients $(21.0 \%$ of all cases), (ii) scan/ ultrasound findings suggesting a multi-gland parathyroid disease in 13 patients (6.1\% of all cases), and (iii) negative scan examination in nine patients $(4.2 \%$ of all cases; two of whom had a cystic parathyroid adenoma and three of whom had a concomitant large multinodular goiter). In this group of 67 patients the probe was utilized during a traditional BNE.

\section{Preoperative imaging procedures}

Planar scintigraphy was performed using a technique developed in our center (14) and consisted of the following steps: acquisition - (i) $150 \mathrm{MBq}{ }^{99 \mathrm{~m}} \mathrm{Tc}$-pertechnetate were injected i.v., (ii) $20 \mathrm{~min}$ later, $400 \mathrm{mg}$ potassium perchlorate were orally administered, (iii) immediately after potassium perchlorate administration, the patient's neck was immobilized under the gamma camera and a 5 min thyroid scan was acquired, (iv) $550 \mathrm{MBq}$ MIBI were injected i.v., followed by $30 \mathrm{ml}$ saline with the aim of avoiding radiotracer stagnation, (v) a dynamic acquisition of seven 5 min MIBI scans was obtained; and processing - (vi) background activity was subtracted from ${ }^{99 \mathrm{~m}}$ Tc-pertechnetate and MIBI scans, (vii) normalization of MIBI scans to the maximum pixel count activity of the ${ }^{99 \mathrm{~m}} \mathrm{Tc}$-pertechnetate scan was performed, (viii) the ${ }^{99} \mathrm{~m}$ Tc-pertechnetate scan was subtracted from the MIBI scan (an interactive software program capable of subtracting incremental fractions of the ${ }^{99 \mathrm{~m}} \mathrm{Tc}$-pertechnetate activity was utilized). 
Potassium perchlorate was given with the purpose of speeding up ${ }^{99 \mathrm{~m}} \mathrm{Tc}$-pertechnetate wash-out from the normal thyroid gland, thus obtaining early high-quality MIBI scans to highlight also parathyroid adenomas located behind the thyroid gland (Figs 1-3). Scans were interpreted by two nuclear medicine physicians skilled in parathyroid imaging; in cases of discrepancy, the final scintigraphic diagnosis was reached by consensus. In patients with a ${ }^{99 \mathrm{~m}}$ Tc-pertechnetate and ultrasound 'normal' thyroid gland, a single focus of MIBI uptake in the MIBI scans and subtraction scans was defined as solitary parathyroid adenoma, while patients with at least two foci of MIBI uptake were considered as being affected by multi-gland disease. In patients with concomitant nodular goiter, correlative ultrasound imaging was used to distinguish parathyroid enlargements from MIBI-avid thyroid nodules.

Planar scintigraphy was acquired by a large-field-ofview gamma camera (Orbiter 7500; Siemens, Hoffman Estates, IL, USA) equipped with a parallel-hole, lowenergy, high-resolution collimator. Images were stored in a $128 \times 128$ matrix and processed using a dedicated computer (ICON-P; Siemens).

SPECT acquisition was performed by a dual-head gamma camera (Axis; Picker International, Cleveland, $\mathrm{OH}$, USA) equipped with a parallel-hole low-energy ultra-high-resolution collimator. The following parameters were adopted: elliptical orbit, 120 steps, 30 s per step, $64 \times 64$ matrix. Images were reconstructed using a low-pass filter, cut-off 0.2 , order 5.0-6.0, and processed using a dedicated computer (Odyssey 830 Digital, Maynard, MA, USA). 3-D analysis was also obtained.

In the subgroup of 36 patients in whom a SPECT acquisition was obtained following a positive planar scintigraphy, SPECT suggested a deep location of the parathyroid enlargement in 14 patients and in these cases a parathyroid adenoma was found at surgery in the para-tracheal/para-esophageal space (ten in the neck, four in the upper mediastinum).
A high-resolution $10 \mathrm{MHz}$ transducer was utilized for ultrasound examination (Technos, Esaote, Italy). Longitudinal and transverse neck scans were obtained from the angle of the mandible to the sternal notch. The enlarged parathyroid gland was identified on grayscale imaging by the characteristic appearance of a hypo-echoic nodule distinct from the thyroid gland.

\section{Intraoperative probe-guided procedure}

The MIRS procedure we are currently using was developed in our center in 1999 and the first patients were operated on in September 1999. Before that time, our surgeons performed a scan-direct UNE as previously described (11). The MIRS protocol consisted of the following steps: (i) blood samples were drawn from a peripheral vein during anesthesia induction, just before skin incision and $10 \mathrm{~min}$ after removal of the parathyroid adenoma to measure quick PTH levels, (ii) $37 \mathrm{MBq}$ MIBI were injected i.v. in the operating theatre a few minutes before the start of surgery the injection was followed by a flush of $30 \mathrm{ml}$ saline to avoid radiotracer stagnation, (iii) prior to the surgical incision, the patient's neck was scanned with an $11 \mathrm{~mm}$ gamma probe to individualize the maximum count activity area corresponding to the parathyroid adenoma, (iv) a middle neck access at the level of the sternal notch was utilized, (v) the probe was inserted through a $2-2.5 \mathrm{~cm}$ skin incision after strap muscle retraction, (vi) the probe guided the surgeon to the maximum count activity area corresponding to the parathyroid adenoma (in some patients with a parathyroid adenoma located deep in the neck, a ligature of the middle thyroid vein and sometimes of the inferior thyroid artery was necessary to better visualize the adenoma), (vii) radioactivity was measured on the parathyroid adenoma, thyroid gland and background, (viii) the parathyroid adenoma was removed using microsurgery instruments, (ix) radioactivity was
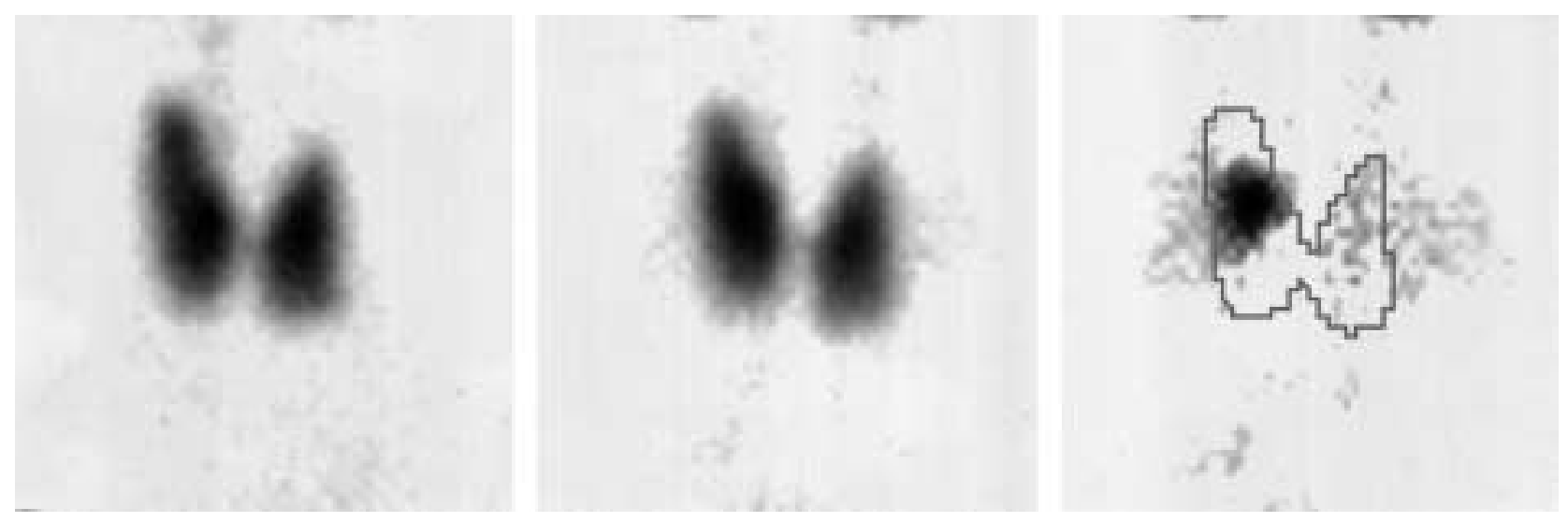

Figure $1^{99 m}$ Tc-pertechnetate scan (left image) shows a normal thyroid gland. MIBI scan (middle image) and subtraction image (right image) show a right superior solitary parathyroid adenoma located behind the thyroid lobe. The patient was treated by MIRS (adenoma weight $=840 \mathrm{mg}$ ). 

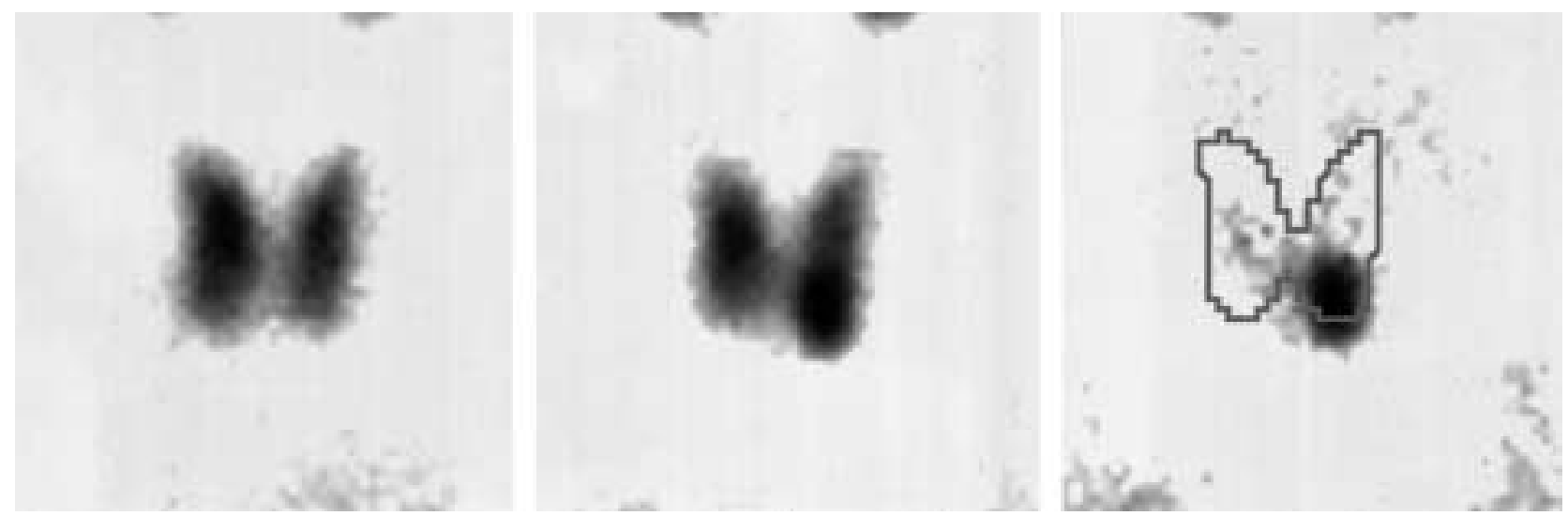

Figure $2{ }^{99 \mathrm{~m}}$ Tc-pertechnetate scan (left image) shows a normal thyroid gland. MIBI scan (middle image) and subtraction image (left image) show a left inferior solitary parathyroid adenoma located partially beyond the thyroid contour. The patient was treated by MIRS (adenoma weight $=1270 \mathrm{mg}$ ).
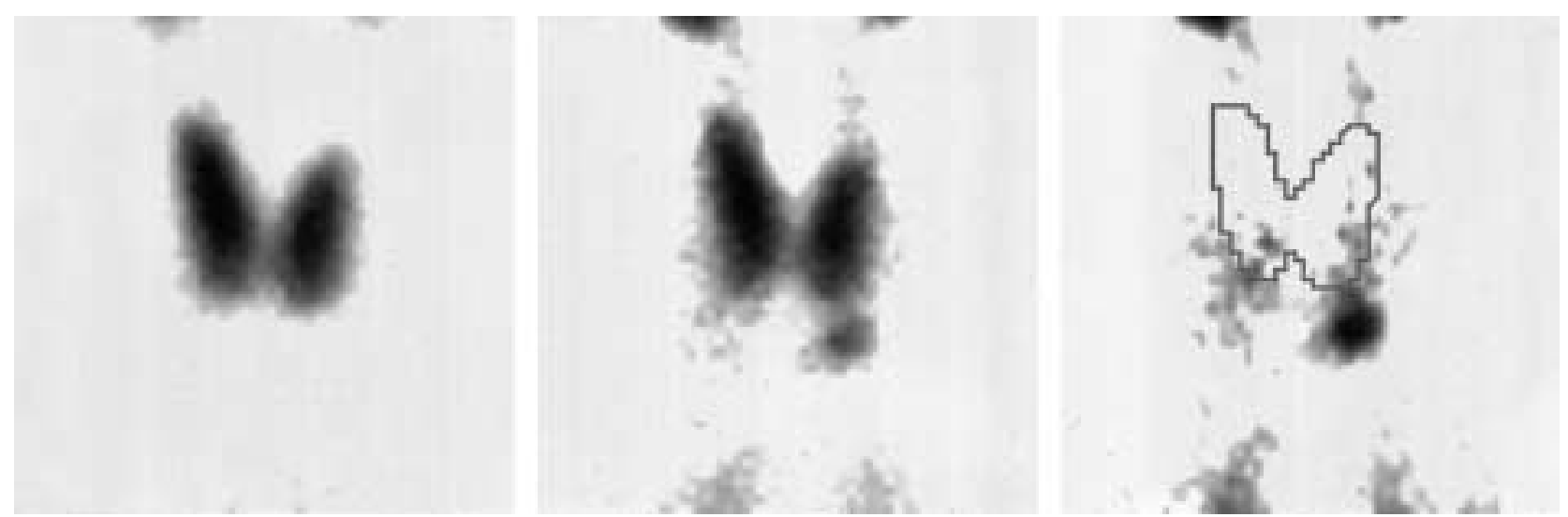

Figure $3^{99 \mathrm{~m}}$ Tc-pertechnetate scan (left image) shows a normal thyroid gland. MIBI scan (middle image) and subtraction image (right image) show a left inferior solitary parathyroid adenoma located beyond the thyroid lobe and well separated from the thyroid contour. The patient was treated by MIRS (adenoma weight $=590 \mathrm{mg}$ ).

checked on the 'ex vivo' parathyroid adenoma as well as on the empty parathyroid bed, $(\mathrm{x})$ tissue ratios were calculated.

In patients with concomitant MIBI-avid nodular goiter, the radioactivity was intraoperatively measured also on the thyroid nodules.

A hand-held commercially available gamma collimated probe (Scintiprobe 100; Pol.hi.tech., Italy) was used. Technical characteristics are as follows: linear probe with an external diameter of $11 \mathrm{~mm}$, mounting a shielded NaI detector; energy range 30-385 keV; gamma detecting efficiency of $99 \%$ for ${ }^{99 \mathrm{~m}} \mathrm{Tc}$ peak energy $(140 \mathrm{keV})$; sensitivity threshold of $10 \mathrm{nCi}$ and spatial resolution of $5 \mathrm{~mm}$ at a $2 \mathrm{~mm}$ distance.

Intraoperative quick PTH was measured by chemoluminescence assay (Liason, Byk Gulden, Italy - normal value $10-54 \mathrm{pg} / \mathrm{ml}$ ). A fall of $50 \%$ or more in PTH levels $10 \mathrm{~min}$ after the parathyroid adenoma removal in comparison with the baseline pre-excision value was considered as indicative of successful parathyroidectomy.
Additional blood samples for quick PTH measurement were obtained in patients with multi-gland disease $10 \mathrm{~min}$ after the removal of all hyperfunctioning parathyroid tissue.

All patients were operated on by the same surgical team. Time from preoperative scan/ultrasound imaging to surgery ranged from 1 to 21 days; in the majority of cases this time interval was $>3$ days. In only four cases was surgery performed the day after scintigraphy; in these patients no significant radioactivity was detected in the neck by the probe before the injection of the $37 \mathrm{MBq}$ MIBI dose required for MIRS.

Post-surgical follow-up ranged from 3 to 34 months, mean \pm S.D $7.8 \pm 5.6$ months.

After surgery, patient received clinical and laboratory surveys 1 month later and subsequently every $2-3$ months.

Data are expressed as means \pm 1 S.D. Mean values were compared using Student's $t$-test. $P$ values $<0.05$ were considered significant. 


\section{Results}

\section{Patients selected for MIRS $(n=147)$}

One hundred and forty-four patients from this group (97.9\% of cases) were successfully treated by MIRS. The probe was particularly helpful in guiding the surgeon to detect 12 ectopic parathyroid adenomas in the upper mediastinum and one at the carotid bifurcation, and ten parathyroid adenomas located deep in the neck in the para-esophageal/para-tracheal space. MIRS required a mean operating time of $35 \mathrm{~min}$ (range 15-58) and a mean hospital stay of 1.2 days (range 1-2). Local anesthesia was performed in 14 patients. No case of laryngeal nerve palsy or permanent hypoparathyroidism was recorded. Transient hypoparathyroidism was observed in $9 \%$ of cases. From a cosmetic point of view, a small $2 \mathrm{~cm}$ skin incision was usually sufficient to perform a MIRS (against the large $>4 \mathrm{~cm}$ skin incision necessary to perform a traditional BNE). In all patients but one, quick PTH levels decreased $>50 \%$ in comparison with the pre-excision value $10 \mathrm{~min}$ after the adenoma removal. Conversion to BNE was required in only three patients $(2.1 \%$ of cases). In one of these three patients quick PTH levels remained elevated after removal of the preoperatively visualized parathyroid adenoma, and another parathyroid adenoma was found at surgery in the contralateral side of the neck (final diagnosis was of double adenoma). In the other two patients a parathyroid carcinoma was diagnosed during intervention and a subtotal thyroidectomy was added to the parathyroidectomy: no loco-regional metastatic spread was found.

Of note, the probe allowed us to perform a MIRS also in 18 out of the 27 patients who had received previous thyroid/parathyroid surgery in another surgical center.

All patients of this group were eucalcemic and had PTH levels within the normal range during the post-surgical follow-up (range 3-34 months, mean 8.0 \pm 5.7 months).

\section{Patients selected for BNE $(n=67)$}

The probe was of little help in this patient group. In particular, in the subgroup of patients with multigland disease the probe helped the surgeon in detecting one ectopic parathyroid gland located in the thymus in one patient; in the subgroup of 45 patients with concomitant nodular goiter the probe was useful to detect an ectopic parathyroid adenoma located at the carotid bifurcation in one patient, and a parathyroid adenoma located deep in the para-esophageal/para-tracheal space in four other patients. However, it has to be pointed out that in some other patients with a parathyroid adenoma located near to the nodular goiter it was difficult to intraoperatively distinguish the radioactivity of the parathyroid adenoma form that of the MIBI-avid thyroid nodules, especially in cases in whom the MIBI uptake was higher in the thyroid nodules than in the parathyroid adenoma. Lastly, in our experience the probe was not useful in the subgroup of patients with a negative preoperative MIBI scan. No case of laryngeal nerve palsy was recorded in patients treated by BNE, while two cases of recurrent HPT (hyperplasia in both cases, in one case MEN-related) were observed during the subsequent follow-up. The rate of transitory hypocalcemia was higher than in patients treated by MIRS (26\%) and, as expected, also the time of intervention was longer (mean $58 \mathrm{~min}$, range $32-90$ ) as well as the hospital stay (mean 2.5 days, range $2-3$ ).

Quick PTH levels reduced $>50 \%$ in all cases after removal of all detected enlarged parathyroid glands. All patients but two were eucalcemic during the post-surgical follow-up (range 3-29 months, mean $7.5 \pm 6.1$ ). Of note, during operation, quick PTH showed a decrease $>50 \%$ also in the two patients who presented recurrent HPT during follow-up.

The size of removed solitary parathyroid adenomas did not significantly differ between patients treated by MIRS and those treated by BNE (mean weight $915 \pm 293$ vs $976 \pm 343 \mathrm{mg}$ respectively). Parathyroid glands were smaller in patients with multi-gland disease $(564 \pm 276 \mathrm{mg} ; P<0.05)$.

We did not perform a randomized study to compare MIRS with traditional BNE in patients with a solitary parathyroid adenoma. However, considering the historical data of our surgical center, both the operating time and the hospital stay were approximately doubled with BNE rather than MIRS.

The radiation exposure dose (calculated in patients who had scintigraphy at least 2 days before surgery) was $1.20 \mu \mathrm{Sv} / \mathrm{h}$ to the surgeon's body and $5.00 \mu \mathrm{Sv} / \mathrm{h}$ to the surgeon's hands, which is significantly lower than the annual radiation dose limits recommended for the general population by the International Commission on Radiological Protection, i.e. $1000 \mu \mathrm{Sv}$ for the body and $50000 \mu \mathrm{Sv}$ for the hands.

\section{Discussion}

In a recent survey from the members of the International Association of Endocrine Surgeons (160 surveys) (15) it emerged that in the year $200059 \%$ of surgeons worldwide were using some type of minimally invasive parathyroidectomy: the videoassisted technique was used by $22 \%$ of surgeons, the true endoscopic technique by $12 \%$ and the probeguided technique by $14 \%$; moreover, quick PTH was measured by $68 \%$ of surgeons.

In contrast to BNE, when considering a MIRS, strict inclusion criteria have to be followed, in particular a high probability of a solitary adenoma established on the basis of a MIBI scan and a significant MIBI uptake in the adenoma, while patients with concomitant 
MIBI-avid thyroid nodules, history of familial HPT or MEN or previous neck irradiation should be excluded from MIRS $(10-13,16)$. As a consequence, only a proportion of primary HPT patients, accounting for about $60-70 \%$ of all cases, can be selected for MIRS (10-13, 16). Moreover, while preoperative imaging could be considered unnecessary when performing a BNE, a MIBI scan becomes mandatory in the view of a MIRS. Various types of preoperative scintigraphic techniques are currently used, the most sensitive being the single-tracer dual-phase SPECT and the double-tracer subtraction technique (17-22). The double-tracer technique offers the advantage of being more specific than the dual-phase technique because it allows diagnosis of thyroid nodular disease concomitant with parathyroid tumors. For this reason the double-tracer scan is usually preferred in geographic areas with a high prevalence of nodular goiter $(21,23)$. We utilize a double-tracer subtraction scintigraphy using ${ }^{99 \mathrm{~m}} \mathrm{Tc}$-pertechnetate as a thyroid agent, which is significantly cheaper than ${ }^{123} \mathrm{I}(14)$. Moreover, a correlative ultrasound imaging is useful to differentiate thyroid from parathyroid enlargements $(21,23)$.

Regarding the intraoperative probe techniques, three principal protocols have been described. The first one was developed by Norman in 1997 (7) consisting of a single-day imaging and surgery approach: the patient is injected with a $740 \mathrm{MBq}$ dose of MIBI, scintigraphy is obtained by dual-phase technique, and within $3 \mathrm{~h}$ from MIBI injection the patient is operated on. Norman's protocol is very attractive, especially from a cost-effectiveness point of view because imaging and surgery are obtained on a single-day basis and, thus, the same MIBI dose is utilized for both imaging and MIRS. A different-day imaging and surgery protocol was proposed by Flynn et al. (24): a MIBI scan is obtained by a dual-phase technique and, usually the day after, the patient is re-injected with an additional high (740 MBq) MIBI dose 60-90 min before surgery. The protocol we developed consists of a different-day imaging and surgery protocol: the scintigraphic examination is obtained by a ${ }^{99 \mathrm{~m}}$ Tc-pertechnetate/MIBI subtraction technique and some days later, for the purpose of probe-guided surgery only, the patient is re-injected with a low (37 MBq) MIBI dose in the operating theatre a few minutes before the start of surgery. The 'low MIBI dose protocol' we propose provides two advantages. First, the radiation exposure dose to both the patient and the surgeon is minimized. Secondly, potential false-negative results due to parathyroid adenomas characterized by a relatively fast MIBI wash-out can be avoided. Furthermore, we prefer using a differentday protocol with the aim of better planning the operating daily program: extension of surgery, time per intervention, number of patients scheduled per day. Thus, even if the single-day protocol of Norman (7) can be considered as the ideal protocol when planning a MIRS, a different-day protocol is probably preferable in geographic areas with a high prevalence of nodular goiter, in order to better select the patients. Again, while in a single-day protocol the injection of a high $740 \mathrm{MBq}$ MIBI dose is required to obtain a scan before surgery, in a different-day protocol a low $37 \mathrm{MBq}$ MIBI dose appears to be adequate to perform a probe-guided surgery (25); thus when using a different-day protocol it does not appear justifiable to re-inject the patient with an additional high $740 \mathrm{MBq}$ MIBI dose.

The main advantages derived by using the gamma probe in our experience were: (i) a rapid intraoperative detection of parathyroid adenomas, especially those located in an ectopic site or deep in the neck, (ii) the evaluation of surgical completeness by checking radioactivity in the empty parathyroid bed after adenoma removal, and (iii) the evaluation of surgical effectiveness by measuring radioactivity on the ex vivo parathyroid adenoma. A quantitative analysis of the $e x$ vivo radioactivity measured on the removed parathyroid tissue has been proposed by Murphy \& Norman (26). Every surgical specimen measuring more than $20 \%$ of background activity was defined as a solitary parathyroid adenoma: applying this parameter, named the ' $20 \%$ rule', the authors found a $100 \%$ accuracy in distinguishing solitary adenomas from multi-gland disease in a very large series of 345 HPT patients. Based on these data, the authors concluded that the performing of frozen section analysis and measurement of quick PTH were unnecessary. These exciting results were not confirmed in other studies (16). Moreover, in our experience the $e x$ vivo radioactivity counting was higher than $40 \%$ in all cases of solitary parathyroid adenoma but also in at least one (dominant) of the enlarged parathyroid glands removed in patients with multi-gland disease. One possible explanation for this discrepancy might be the different timing of MIBI injection and probe measurements: $2-3 \mathrm{~h}$ in Norman's protocol $(7,26)$ but $20-30 \mathrm{~min}$ in our protocol. In this regard, as first demonstrated by O'Doherty et al. in 1992 (18), MIBI reaches the maximum concentration in the parathyroid adenoma within a few minutes from injection; then a slow wash-out is usually observed. Thus, it can be concluded that the ' $20 \%$ rule' is strictly dependent on the type of protocol utilized and can probably only be applied to the Murphy \& Norman protocol (26). Furthermore, in our experience quick PTH was very helpful in some patients with multi-gland disease in whom one enlarged parathyroid gland showed a high probe count rate (dominant) while the other enlarged glands were characterized by a relatively low probe count rate; this finding could lead the surgeon to underestimate multi-gland disease while the persistence of elevated quick PTH levels induces the surgeon to continue exploration. Lastly, in a recent study of Jaskowiak et al. (27), performed on 54 primary HPT patients treated by probe-guided surgery, the 
'20\% rule' was efficacious in identifying all solitary parathyroid adenomas (100\% sensitivity). Unfortunately, the authors did not obtain data about patients with multi-gland disease. However, interestingly they observed that the application of merely the ' $20 \%$ rule' gave false-positive results in the majority of patients with concomitant thyroid nodules (six of seven cases, $85.7 \%$ ) thus heavily affecting specificity. Of note, the authors utilized a dual-phase MIBI technique for the preoperative scan that is recognized as being inaccurate in distinguishing parathyroid adenomas from MIBIavid thyroid nodules; in these cases a double-tracer scintigraphy would be preferred $(21,23)$ and probeguided surgery should be avoided as confirmed by the results of our study. For the above-cited reasons, at the moment, when performing a MIRS, it appears reasonable to recommend a quick PTH measurement with the aim of further evaluating the completeness of all hyperfuctioning parathyroid tissue removal.

It has to be pointed out that in our experience MIRS was successfully performed also in a high percentage of patients (18 of $27,66.6 \%$ ) who had previous thyroid/parathyroid surgery, thus minimizing the risks of possible intraoperative complications. Similar favorable results have been recently reported by Norman \& Denham (28) in re-operative HPT patients. Of note, in contrast to probe-guided surgery, endoscopic surgery has not been proposed for re-operation (29).

A technical aspect to be discussed concerns the utilization of collimated or not collimated probes. Perrier et al. (16) reported favorable results using a non-collimated probe. However, collimated probes offer the advantage of avoiding or minimizing the interfering radioactivity from the surrounding tissues, especially from the thyroid gland (25).

From a surgical point of view, we prefer using a middle neck access mainly because conversion to a BNE can be easily performed when necessary. Using a middle access, MIRS was successfully performed in $98 \%$ of patients in our series. However, it is worth noting that other authors reported similarly good results using a lateral neck access $(7,24,26,30)$. Probably this issue strictly depends on the surgeon's own experience and convenience.

In our study the probe was not helpful in primary HPT patients with a negative preoperative MIBI scan. This is in contrast to the experiences of Bonjer et al. (31) and Jaskowiak et al. (27) who claimed that a major role of the gamma probe was the intraoperative detection of unknown (scan-negative) hyperfunctioning parathyroid glands during BNE. This discrepancy might be explained, at least in part, by considering the relatively lower sensitivity of the preoperative scintigraphy used by Bonjer et al. (31) and Jaskowiak et al. (27) (dual-phase technique) in comparison with our scintigraphic procedure (double-tracer technique): sensitivity for detection of parathyroid adenomas was $60-74 \%$ vs $95 \%$ respectively.
It appears surprising that such different and opposite opinions have been reported in the literature on the role of the gamma probe during parathyroidectomy, ranging from enthusiastic considerations $(7,26,28)$, to less favorable results $(16,27)$, to a judgment of uselessness (32). Some explanations can be made. Interestingly, Jaskowiak et al. (27), proposed a four-point scale to define the probe-guided surgery in a group of 54 patients treated by BNE: the probe was judged crucial in only two patients (4\%), helpful in $12(22 \%)$, merely confirmatory in $32(59 \%)$ and not helpful in eight $(15 \%)$. In contrast, in our experience as well as in that of Norman et al. $(7,26,28)$ the probe was helpful in the majority of scan-positive patients and very helpful or even crucial in patients with adenomas located in ectopic sites or deep in the para-tracheal/ para-esophageal space. This difference in judging probe utility could be related, at least in part, to the different surgical approaches utilized: a BNE in Jaskowiak's study (27) while a MIRS in our own study and in Norman's studies $(7,26,28)$. From this view, it appears reasonable to think that it can be relatively easy for an experienced surgeon to detect a parathyroid adenoma located in an orthotopic site during a BNE simply using the 'eyes'; conversely, it can be difficult, even for an experienced surgeon, to detect a parathyroid adenoma through a $2 \mathrm{~cm}$ skin incision without the help of a 'gamma probe': thus, following Jaskowiak's criteria (27) what is judged as 'merely confirmatory' during a BNE could be 'helpful' during a MIRS and what is judged as 'helpful' during a BNE could be 'crucial' during a MIRS.

Debate exists about the cost-effectiveness analysis of probe-guided surgery $(7,10-13,16,24-30)$. It depends upon various factors: (i) when a MIRS is planned, a preoperative MIBI scan is necessary, (ii) the single-day protocol takes advantage of using a single MIBI dose for preoperative imaging and probe-guided surgery, (iii) in the case of a different-day imaging and surgery protocol two doses of MIBI are required, (iv) the purchase of the gamma probe and connected electronic devices, and (v) the ability or not to perform also frozen section analysis and quick PTH measurement. All of these items have a cost that can, however, vary from country to country and depends also upon the local care rules and objectives. In our surgical center the gamma probe was already available when we started performing MIRS, since is was used for sentinel node biopsy of melanoma and breast cancer patients. Moreover, in our nuclear medicine unit MIBI is utilized on a daily basis for various types of scintigraphic examinations such as myocardial, breast and thyroid cancer scans (33) and consequently the cost of a MIBI dose as low as $37 \mathrm{MBq}$ to perform a MIRS is essentially negligible. Regarding quick PTH, its measurement has been used in our center for many years and is currently utilized by our surgeons independent of the type of parathyroid surgery they plan, 
i.e. during both BNE and MIRS. Most importantly, some of the principal objectives of our hospital administration are to reduce operating time, increase the daily number of patients scheduled for intervention, and reduce the hospital stay: in our experience the utilization of the gamma probe facilitated the reaching of these objectives in primary HPT patients.

It can be concluded that: (i) in geographic areas with high prevalence of nodular goiter, the combination of MIBI scan, performed by double-tracer technique, and neck high-resolution ultrasound is an accurate protocol in selecting primary HPT patients as candidates for a probe-guided minimally invasive parathyroidectomy, also in patients who need re-operation, (ii) when using a different-day imaging and surgery protocol a MIBI dose as low as $37 \mathrm{MBq}$ appears to be adequate for the purpose of MIRS, (iii) with the possible exception of parathyroid adenomas located deep in the neck or in an ectopic site, probe-guided surgery does not appear recommendable in patients with concomitant nodular goiter, and (iv) at the moment, it appears recommendable to measure quick PTH levels during MIRS with the aim of further evaluating completeness of all hyperfunctioning parathyroid tissue removal.

\section{Acknowledgements}

This paper was presented by invitation at the Royal College of Surgeons Meeting, Parathyroid Localisation - Utility of Diagnostic Imaging, held in London on 22 November 2002.

\section{References}

1 Kaplan E, Yashiro T \& Salti G. Primary hyperparathyroidism in the 1990s: choice of surgical procedures for this disease. Annals of Surgery $1992 \mathbf{2 1 5}$ 300-317.

2 Kaplan E, Barlett S, Sugimoto J \& Fredland A. Relation of postoperative hypocalcemia to operative technique: deleterious effect of excessive use of parathyroid biopsy. Surgery 198292 $827-834$.

3 Feingold DL, Alexander HR, Chen CC, Lubutti SK, Shawker TH, Simonds WF et al. Ultrasound and sestamibi as the only preoperative imaging tests in reoperation for parathyroid adenomas. Surgery $2000 \mathbf{1 2 8} 1103-1109$.

4 Auguste LJ, Attie JN \& Schnaap D. Initial failure of surgical exploration in patients with primary hyperparathyroidism. American Journal of Surgery $1990160333-336$.

5 Tibblin S, Bondeson AG \& Ljubgberg O. Unilateral parathyroidectomy in hyperparathyroidism due to a single adenoma. Annals of Surgery 1982195 245-252.

6 Gagner M. Endoscopic parathyroidectomy [letter]. British Journal of Surgery $1996 \mathbf{8 3} 875$.

7 Norman J \& Chheda H. Minimally invasive parathyroidectomy facilitated by intraoperative nuclear mapping. Surgery 1997 122 998-1004.

8 Coakley AJ, Kettle AG, Wells CP, O'Doherty MJ \& Collings REC. 99 mTc-sestamibi - a new agent for parathyroid imaging. Nuclear Medicine Communications $198910791-794$.

9 Irvin GL, Dembrow VD \& Prudhomme DL. Clinical usefulness of an intraoperative 'quick PTH' assay. Surgery $1993 \mathbf{1 1 4}$ $1019-1023$
10 Casara D, Rubello D, Piotto A \& Pelizzo MR. 99mTc-MIBI radioguided minimally invasive parathyroid surgery planned on the basis of a preoperative combined $99 \mathrm{mTc}$-pertechnetate $/ 99 \mathrm{mTc}$ MIBI and ultrasound imaging protocol. European Journal of Nuclear Medicine 200027 1300-1304.

11 Casara D, Rubello D, Pelizzo MR \& Shapiro B. Clinical role of $99 \mathrm{mTcO}_{4} / \mathrm{MIBI}$ scan, ultrasound and intra-operative gamma probe in the performance of unilateral and minimally invasive surgery in primary hyperparathyroidism. European Journal of Nuclear Medicine 2001 28 1351-1359.

12 Casara D, Rubello D, Cauzzo C \& Pelizzo MR. 99mTc-MIBI radioguided minimally invasive parathyroidectomy: experience with patients with normal thyroids and nodular goiters. Thyroid $20021253-61$.

13 Rubello D, Casara D, Giannini S, Piotto A, DeCarlo E, Muzzio PC et al. Importance of radio-guided minimally invasive parathyroidectomy using hand-held gamma probe and low $99 \mathrm{mTc}-\mathrm{MIBI}$ dose: technical considerations and long-term clinical results. Quarterly Journal of Nuclear Medicine 200347 (In Press).

14 Rubello D, Saladini G, Casara D, Piotto A, Toniato A, Bernante P et al. Parathyroid imaging with pertechnetate plus perchlorate/ MIBI subtraction scintigraphy. A fast and effective technique. Clinical Nuclear Medicine 200025 527-531.

15 Sackett WR, Barraclough B, Reeve TS \& Delbridge LW. Worldwide trends in the surgical treatment of primary hyperparathyroidism in the era of minimally invasive parathyroidectomy. Archives of Surgery 2002137 1055-1059.

16 Perrier ND, Ituarte PHG, Morita E, Hamill T, Gielow R, Duh Q-Y et al. Parathyroid surgery: separating promise from reality. Journal of Clinical Endocrinology and Metabolism 200287 1024-1029.

17 Taillefer R, Boucher Y, Potvin C \& Lambert R. Detection and localization of parathyroid adenomas in patients with hyperparathyroidism using a single radionuclide imaging procedure with technetium-99m-sestamibi (double-phase study). Journal of Nuclear Medicine 199233 1801-1807.

18 O’Doherty MJ, Kettle AG, Wells P, Collins REC \& Coakley AJ. Parathyroid imaging with technetium-99m-sestamibi: preoperative localization and tissue uptake studies. Journal of Nuclear Medicine 199233 313-318.

19 Hindié E, Melliere D, Jeanguillaume C, Perlemuter L, Chehade F \& Galle P. Parathyroid imaging using simultaneous double-window recording of technetium-99m-sestamibi and iodine-123. Journal of Nuclear Medicine $1998391100-1105$.

20 Sfakianakis GN, Irvin GL, Foss J, Mallin W, Georgiou M, Derosi GT et al. Efficient parathyroidectomy guided by SPECT-MIBI and hormonal measurements. Journal of Nuclear Medicine 199637 798-804.

21 Rubello D, Casara D \& Shapiro B. Recent advances in preoperative and intraoperative nuclear medicine procedures in patients with primary hyperparathyroidism. Panmiverva Medica $2002 \mathbf{4 4}$ 99-105.

22 Coakley AJ. Symposium on parathyroid localization [editorial]. Nuclear Medicine Communications 200324 111-113.

23 O'Doherty MJ \& Kettle AG. Symposium on parathyroid localization. Parathyroid imaging: preoperative localization. Nuclear Medicine Communications 200324 125-131.

24 Flynn MB, Bumpouns JM, Schill K \& McMasters KM. Minimally invasive radioguided parathyroidectomy. Journal of the American College of Surgeons $200019124-31$.

25 Rubello D, Casara D \& Pelizzo MR. Symposium on parathyroid localising imaging. Optimisation of peroperative procedures. Nuclear Medicine Communications 200324 133-140.

26 Murphy C \& Norman J. The 20\% rule: a simple, instantaneous radioactivity measurement defines cure and allows elimination of frozen sections and hormone assays during parathyroidectomy. Surgery 1999126 1023-1029.

27 Jaskowiak NT, Sugg SL, Helke J, Kota MR \& Kaplan EL. Pitfalls of intraoperative quick parathyroid hormone monitoring and gamma probe localization in surgery for primary hyperparathyroidism. Archives of Surgery 2002137 659-669. 
28 Norman J \& Denham D. Minimally invasive radioguided parathyroidectomy in the reoperative neck. Surgery $1998 \mathbf{1 2 4} 1088-1093$.

29 Rubello D, Pelizzo MR \& Casara D. Nuclear medicine and minimally invasive surgery of parathyroid adenomas: a fair marriage [editorial]. European Journal of Nuclear Medicine 200230 189-192.

30 McGreal G, Winter DC, Sookhai S, Evoy D, Ryan M, O'Sullivan GC et al. Minimally invasive, radioguided surgery for primary hyperparathyroidism. Annals of Surgical Oncology 2001 8 856-860.

31 Bonjer HJ, Bruining HA, Pols HAP, de Herder WW, van Eijck CHJ, Breeman WAP et al. Intraoperative nuclear guidance in benign hyperparathyroidism and parathyroid cancer. European Journal of Nuclear Medicine $1997 \mathbf{2 4} 246-251$.
32 Inabnet WB III, Kim CK, Haber RS \& Lopchinsky RA. Radioguidance is not necessary during parathyroidectomy. Archives of Surgery $2002137967-970$.

33 Rubello D, Mazzarotto R \& Casara D. The role of technetium-99m methoxyisobutylisonitrile scintigraphy in the planning of therapy and follow-up of patients with differentiated thyroid carcinoma. European Journal of Nuclear Medicine 200027 431-440.

Received 2 December 2002

Accepted 2 April 2003 